\title{
An Interventional Quality Improvement Study to Assess the Compliance to Cardiopulmonary Resuscitation Documentation in an Indian Teaching Hospital
}

\author{
Viraj Nevrekar, Prasan Kumar Panda, Naveet Wig, R. M. Pandey', Praveen Agarwal2, Ashutosh Biswas \\ Departments of Internal Medicine, ${ }^{1}$ Biostatistics and ${ }^{2}$ Emergency Medicine, All India Institute of Medical Sciences, New Delhi, India
}

Abstract

Background: Cardiopulmonary resuscitation (CPR) should be performed as per the international guidelines; however, compliance to these guidelines is difficult to assess. This study was conducted to determine the compliance to American Heart Association (2010) guideline on CPR documentation by among resident physicians before and after resident training (two arms). Methods: This pre-postinterventional quality improvement study was conducted in a referral center, North India. Data of hospitalized in-hospital CPR patients were collected in the form of quality indicators (checklists) as defined by the guideline and compared between two arms of before-after resident training. Residents were given appropriate training in CPR technique as per the guideline. The compliance of CPR documentation was assessed pre- and post-intervention. Results: The baseline arm compliance of various components of CPR documentation was low. The postintervention arm compliances of all components significantly increased (baseline, $2.5 \%$ to postintervention, $15.11 \%, P=0.03$ ). Individual components assessed were documentation of assessment of responsiveness $(65 \%$ to $77.9 \%, P=0.19)$, assessment of breathing $(37.5 \%$ to $58.1 \%, P=0.03)$, assessment of carotid pulse $(62.5 \%$ to $79 \%, P=0.05)$, rate of chest compressions $(20 \%$ to $39.5 \%, P=0.04)$, airway management $(62.5 \%$ to $82.5 \%, P=0.02)$, and compressions to breaths ratio $(12.5 \%$ to $31.4 \%, P=0.02)$. Documentation of chest compression rate compared to nondocumentation (12 of 42 vs. 11 of $84, P=0.04$ ) was independently associated with a higher rate of return of spontaneous circulation. The study however did not show any survival benefits. Conclusions: This study establishes that the compliance to CPR documentation is poor as assessed by CPR documentation content and quality, which improves after physician training, but not up to the mark level (100\%) that may be due to busy Indian hospital settings and human behavioral factors. Due to ethical constraints of live CPR assessment, this document checklist approach may be considered as an internal quality assessment method for CPR compliance. Furthermore, correct instruction in CPR technique along with proper documentation of the procedure is required, followed up with periodic re-education during the residency period and beyond.

Keywords: Adults, cardiopulmonary resuscitation documentation, in-hospital cardiac arrest, quality appraisal

\section{INTRODUCTION}

Cardiopulmonary resuscitation (CPR) has long been used as the most scientifically approved modality of revival, yet survival of patients postcardiac arrest has been low. ${ }^{[1-3]}$ Although the guidelines define how a resuscitation is to be performed, neither are the CPR parameters routinely measured nor is the compliance known. ${ }^{[4]}$ Several studies document that all major components of the resuscitation, i.e., chest compressions, ventilation rates, defibrillation, endotracheal intubation, and administration of intravenous medications, may be performed poorly. ${ }^{[5-8]}$ Incorrect CPR is associated with a 14-day survival rate of $4 \%$ compared to $16 \%$ when CPR is performed correctly. ${ }^{[5]}$ Hence, compliance to the established guidelines is essential.

\begin{tabular}{|l|l|}
\hline \multicolumn{3}{c|}{ Access this article online } \\
\hline Quick Response Code: & Website: \\
& www.ijccm.org \\
\hline
\end{tabular}

For healthcare providers, goals of CPR remain (a) recognize and respond to patients at risk of cardiac arrest, (b) deliver quality CPR whenever required, and (c) improve the entire resuscitation process through improved teamwork. ${ }^{\left[{ }^{9]}\right.}$ For a hospital/institute, universal elements of a system of care are required that include structure (e.g., people, equipment,

Address for correspondence: Dr. Naveet Wig, Department of Internal Medicine, All India Institute of Medical Sciences, Ansari Nagar, New Delhi - 110 029, India. E-mail: naveet_wig@yahoo.com

This is an open access article distributed under the terms of the Creative Commons Attribution-NonCommercial-ShareAlike 3.0 License, which allows others to remix, tweak, and build upon the work non-commercially, as long as the author is credited and the new creations are licensed under the identical terms.

For reprints contact: reprints@medknow.com

How to cite this article: Nevrekar V, Panda PK, Wig N, Pandey RM, Agarwal P, Biswas A. An interventional quality improvement study to assess the compliance to cardiopulmonary resuscitation documentation in an Indian teaching hospital. Indian J Crit Care Med 2017;21:758-64. 
and education), process (e.g., policies, protocols, and procedures), system (e.g., programs, organizations, and cultures), and optimal outcomes (e.g., patient survival and safety, quality, and satisfaction) in a framework of continuous quality improvement (QI). To provide high-quality CPR, all these factors need to be considered. Involvement of resident physician in CPR in India is very much considerable.

To deliver high-quality CPR, training programs are almost essential. These learned resuscitation skills deteriorate over time. ${ }^{[10]}$ Therefore, alternative training strategies in addition to the standard courses of resuscitation training should be used. Techniques, such as simulation, automated quantitative feedback during training, postevent debriefing, and regular training, have shown promise. ${ }^{[11-15]}$ However, the American Heart Association (AHA) guidelines do not recommend these methods to improve learning and retention due to insufficient evidence. ${ }^{[11]}$ Furthermore, in India, the effectiveness of training is always questionable due to lack of strong institute policy and of internal quality control assessments.

At last, assessment methods of CPR compliance are not outlined in the guideline, except internal QI programs that indirectly maintain the quality. Majority of Indian hospitals lack this internal quality programs. Due to ethical issues of direct live CPR assessment, indirect methods are there to assess the compliance (e.g., survival outcome analysis). The compliance of post-CPR documentation can be an indirect method of assessment.

Given the above background, we did a QI study (pre-postintervention design with two arms) to know whether training of residents could improve AHA guideline compliance of CPR documentation. We hypothesized that baseline CPR documentation would be highly variable $(<30 \%)$. Further, we hypothesized that booster trainings on CPR by lectures, live demonstrations, and provision of a checklist would result in $>90 \%$ of participants delivering high-quality AHA guideline-compliant CPR with their proper documentation during the posttraining evaluations.

\section{Methods}

\section{Study settings}

The study was conducted in a tertiary care medical institution, Delhi, India, in a period from January 2012 to September 2013 with a primary objective of assessing the documentation content and quality of AHA guideline-compliant CPR after resident physicians' training (second arm) and comparing this to baseline documentation available before the intervention (first arm). The medical college attached hospital offered quality health services including education and research facilities through the hierarchy of doctors (from interns to residents to professors) as well as thorough documentation of the patients' details. However, no other QI project on CPR was going on during that time.

\section{Participants and variables}

The study recruited in-hospital cardiac arrest patients from the medicine wards, emergency ward, and medicine Intensive Care Unit (ICU). Arrests were defined as the absence of a pulse in an unconscious individual with no respiration or with agonal breaths only. ${ }^{[16]}$ Because of ethical issues of observing live CPR without any participation, case records of patients who were resuscitated were collected. Documentation was assessed based on the following six quality indicators [checklists - Figure 1], as defined by the AHA-2010 guidelines: (a) assessment of responsiveness, (b) assessment of breathing, (c) examination of carotid pulse, (d) rate of chest compressions, (e) documentation of airway, and (f) documentation of compressions to breaths ratio. ${ }^{[17]}$

Patients $<12$ years of age, out-of-hospital cardiac arrests, and posttraumatic arrests were excluded from the study. The documentation of 126 cardiac arrest patients (40 in the preintervention phase and 86 in the postintervention phase) were studied. Among investigators, only one was given the task of reviewing all documents to avoid the interobserver variations.

\section{Study phases}

The study was conducted under three phases.

\section{Preintervention phase}

The documentation of 40 CPRs was studied sequentially from the date of enrolment after applying exclusion criteria. The baseline phase lasted for 6 months during which guideline compliance was assessed in the form of six quality indicators via the available documentation through the use of a standardized checklist. Document compliance, an indirect measure of quality of CPR, was defined as the documentation of components of CPR in an order as defined by the AHA-2010 guideline.

\section{Intervention phase (education and training phase)}

This phase lasted for 3 months. Six sessions of the training were conducted covering 50 medicine residents batch by batch with a presession assessment. They were chosen for the intervention/

\begin{tabular}{|l|l|l|}
\hline \multicolumn{2}{|c|}{$\begin{array}{l}\text { Documentation check list for CPR skills } \\
\text { If documented correctly } \\
\text { as per guideline }\end{array}$} \\
\hline 1 & $\begin{array}{l}\text { Critical performance } \\
\text { steps } \\
\text { Documentation on } \\
\text { responsiveness } \\
\text { Documentation on } \\
\text { breathings }\end{array}$ & Yes/No \\
\hline 2 & $\begin{array}{l}\text { Documentation on } \\
\text { carotid pulse } \\
\text { Documentation on } \\
\text { compression rate and } \\
\text { depth } \\
\text { Documentation on } \\
\text { airway status } \\
\text { Documentation on } \\
\text { ventilation rate as per } \\
\text { type of delivery }\end{array}$ & Yes/No \\
\hline 5 & Yes/No \\
\hline 6 & \\
\hline
\end{tabular}

Figure 1: Study checklist used to assess CPR documentation quality. CPR: Cardiopulmonary resuscitation 
training because of their leadership role during the CPR procedure among other staffs (e.g., nurses and technicians) involved in the teamwork. It was presumed that all residents were skilled in CPR procedure as a result of their internship training and experience. They were uninformed about the ongoing study (blinded) to prevent bias of writing a good CPR document despite not being guideline compliant. They were provided with a series of lectures and checklist of processes involved in CPR by the investigators. Simulation-based training sessions were given by one investigator who was advanced cardiac life support certified. Residents were also provided hands-on training in CPR. Feedback was provided to them at the end of the training. However, neither were separate resident records maintained nor were residents monitored individually postintervention.

It is important to note that all residents who attended the training sessions had improved the quality of CPR delivery from $<30 \%$ to $>90 \%$ as assessed immediately before and after the training sessions, thereby demonstrating the adequacy of the training sessions themselves.

\section{Postintervention phase}

The documentations of $86 \mathrm{CPRs}$, performed over 1 year, were studied sequentially after the exclusion. The primary outcome measure was the change in compliance in accordance with AHA-2010 guideline over time. Secondary outcome measures included revival rate or return of spontaneous circulation (ROSC), hospital length of stay, and postresuscitation functional status. ROSC was defined as the return of pulse and its maintenance for at least $20 \mathrm{~min}$. Trained residents were not followed up to avoid a selection bias. Because of long duration of this phase, attrition over time toward the training must had impact on the study.

\section{Statistical analysis}

All data were entered into Microsoft ${ }^{\circledR}$ Excel spreadsheet, and the data were analyzed using SPSS ${ }^{\circledR}$ software version 17.0 (SPSS Statistics for Windows, Version 17.0. SPSS Inc. Chicago). Chi-square test, Fisher's exact test, and unpaired Student's $t$-test were used for the differences between the groups. The logistic regression was used with mortality as the dependent variable and individual bundle quality indicators as the independent variables. A $P<0.05$ was considered statistically significant.

\section{Ethical approval}

The study protocol was approved by the institutional review board. Data collection procedures were completed with ensuring the subject confidentiality.

As part of educational training under the master degree medical curriculum, resident physicians were given training and they were completely blinded from the study concerned.

\section{RESULTS}

\section{Baseline characteristics}

A total of 130 patients' records were screened and 126 patients who met inclusion criteria were taken for the study. Figure 2 shows the study flowchart. Intervention groups were similar in major baseline demographics, including the sex but not age, acute physiology and chronic health evaluation score which includes comorbid illnesses, status of the patients at first responder, interventions/devices attached to the patients, immediate cause of arrests (within $1 \mathrm{~h}$ ) except the respiratory failure, all-cause mortality, reversible factors at the time of arrest, use of defibrillators with types of abnormal rhythms, and use of drugs during CPRs except adrenaline (epinephrine) [Table 1]. Significant age disparity in between two groups was due to more number of enrolled ICU patients (more number of younger patients in medical ICU than ward because of selective inclusion criteria in ICU admission for practical reasons) in the postintervention group. Moreover, ICU patient disparity was due to more number of admitted sick patients in the latter group as par with tropical seasonal variations (July-December caseloads were higher than January-June admissions in our country). The most common cause of death continued to be sepsis in both groups $(>90 \%)$ followed by the coronary event.

\section{Primary outcome variables: Compliance with the cardiopulmonary resuscitation guideline}

The entire CPR protocol compliance of documentation showed a progressive and significant improvement from the preintervention at $2.5 \%$ compliance to postintervention phase at $15.11 \%$ compliance $(P=0.04)$ [Table 2]. Four components showed statistically significant improvement postintervention, including the documentation of assessment of breathing (20.6\% absolute improvement), rate of chest compressions (19.5\%), airway management (20\%), and compressions to breaths ratio (18.9\%). Two other components (assessment of responsiveness, 12.9\% absolute improvement and carotid pulsation, 16.5\%) showed improvement in compliance without any statistical significance. Postintervention assessment also showed that all performed CPRs had at least two documented

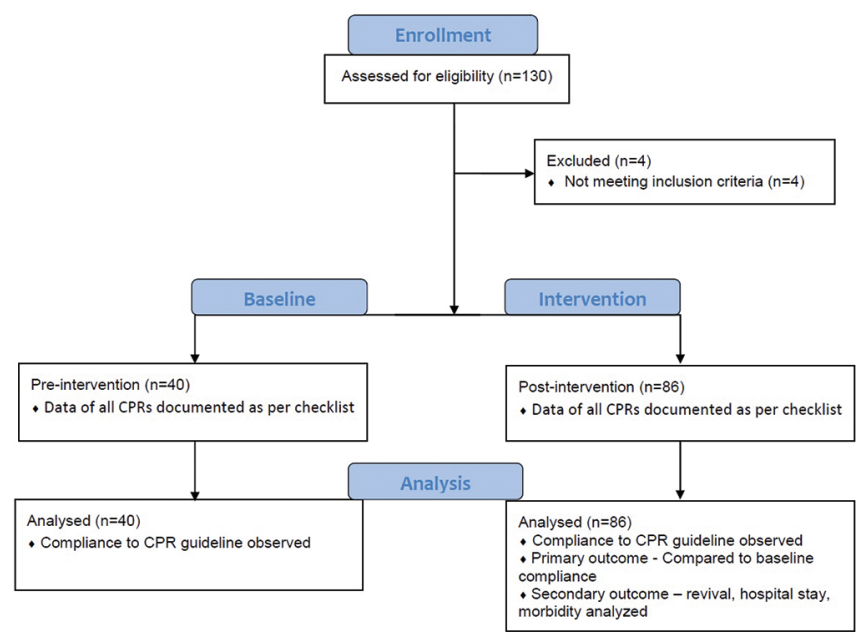

Figure 2: Study design flow sheet. $n$ : Sample number; CPR: Cardiopulmonary resuscitation 


\begin{tabular}{|c|c|c|c|}
\hline Characteristics & Preintervention $(n=40)$ & Postintervention $(n=86)$ & $P$ \\
\hline Sex (female) & $17(42.5)$ & $47(54.6)$ & 0.25 \\
\hline Mean age (years) & $61.8 \pm 13.6$ & $52.9 \pm 19.3$ & 0.004 \\
\hline APACHE II score & $30.9 \pm 9.4$ & $26.8 \pm 9.4$ & 0.46 \\
\hline \multicolumn{4}{|c|}{ Location of the patient at the time of arrest } \\
\hline Emergency ward & $8(20)$ & $22(25.6)$ & 0.65 \\
\hline Medicine ward & $25(62.5)$ & $35(40.7)$ & 0.03 \\
\hline ICU & $7(17.5)$ & $29(33.7)$ & 0.08 \\
\hline \multicolumn{4}{|c|}{ Status of the patient at first respond to arrest } \\
\hline Witnessed, monitored & $23(57.5)$ & $39(45.3)$ & 0.25 \\
\hline Witnessed, not monitored & $8(20)$ & $31(36)$ & 0.09 \\
\hline Monitored, not witnessed & $9(22.5)$ & $14(16.3)$ & 0.45 \\
\hline Not monitored, not witnessed & 0 & $2(2.3)$ & 1.0 \\
\hline \multicolumn{4}{|c|}{ Interventions present at the time of arrest } \\
\hline Vascular access & $37(92.5)$ & $82(95.4)$ & 0.67 \\
\hline ECG monitor & $36(90)$ & $76(88.4)$ & 1.0 \\
\hline Pulse oximeter & $37(92.5)$ & $73(84.9)$ & 0.38 \\
\hline Invasive ventilation & $34(85)$ & $65(75.6)$ & 0.25 \\
\hline Noninvasive ventilation & $8(20)$ & $19(22.1)$ & 1.0 \\
\hline Dialysis & $13(32.5)$ & $32(37.2)$ & 0.69 \\
\hline Chest tube & $10(25)$ & $15(17.4)$ & 0.34 \\
\hline \multicolumn{4}{|c|}{ Immediate cause of cardiac arrest (within $1 \mathrm{~h}$ ) } \\
\hline Toxicological problem & $5(12.5)$ & $11(12.8)$ & 1.0 \\
\hline Arrhythmia & $8(20)$ & $20(23.3)$ & 0.81 \\
\hline Respiratory failure & $8(20)$ & $36(41.9)$ & 0.01 \\
\hline Hypotension & $16(40)$ & $28(32.6)$ & 0.42 \\
\hline Myocardial infarction & $8(20)$ & $18(20.9)$ & 1.0 \\
\hline Pulmonary embolism & $1(2.6)$ & 0 & 1.0 \\
\hline Electrolyte abnormality & $23(57.5)$ & $41(47.7)$ & 0.34 \\
\hline \multicolumn{4}{|l|}{ All-cause mortality } \\
\hline Sepsis & $36(90)$ & $78(90.7)$ & 1.0 \\
\hline Acute coronary event & $3(7.5)$ & $6(6.9)$ & 1.0 \\
\hline Others & $1(2.5)$ & $2(2.4)$ & 1.0 \\
\hline \multicolumn{4}{|c|}{ Reversible factors present at time of arrest } \\
\hline Hypoxia & $30(75)$ & $54(62.7)$ & 0.22 \\
\hline Hypokalemia & $13(32.5)$ & $27(31.3)$ & 1.0 \\
\hline Hypotension & $10(25)$ & $28(32.5)$ & 0.41 \\
\hline Others & $6(15)$ & $10(11.6)$ & 0.57 \\
\hline \multicolumn{4}{|l|}{ Use of defibrillation } \\
\hline First rhythm (asystole) & $34(85)$ & $78(90.6)$ & 0.37 \\
\hline Received defibrillation & $6(15)$ & $8(9.3)$ & 0.37 \\
\hline Revived/ROSC & $5(83.3)$ & $11(78.5)$ & 1.0 \\
\hline Average number of shocks & 1.66 & 2 & - \\
\hline \multicolumn{4}{|c|}{ Drugs administered at time of cardiopulmonary resuscitation } \\
\hline Epinephrine & $1(2.5)$ & $13(15.1)$ & 0.03 \\
\hline Dopamine & $14(35)$ & $27(31.4)$ & 0.68 \\
\hline Norepinephrine & $16(40)$ & $31(36)$ & 0.69 \\
\hline Dobutamine & $10(25)$ & $26(30.2)$ & 0.67 \\
\hline Vasopressin & 0 & $1(1.2)$ & 1.0 \\
\hline Lidocaine & 0 & $3(3.5)$ & 0.55 \\
\hline Amiodarone & $3(7.5)$ & $5(5.8)$ & 0.78 \\
\hline
\end{tabular}

Figures in bracket indicate percentage (\%). APACHE: Acute Physiology and Chronic Health Evaluation; ICU: Intensive Care Unit; ROSC: Return of spontaneous circulation; ECG: Electrocardiography

components and majority had documentation of 3-4 components. It thus showed an overall improvement in the compliance.

\section{Secondary outcome variables}

Out of 126 patients enrolled, 23 (18.25\%) had ROSC and $8(6.35 \%)$ survived till the discharge. Postintervention 
rate of ROSC did not show any significant improvement ( $1.1 \%$ absolute increments) [Table 3]. Hospital stay also did not show significant difference in between two compared groups. All patients who survived until the discharge had moderate to good cerebral function.

On subgroup analysis of CPR components among the revived patients, documentation of assessment of responsiveness and of chest compression rate ( $P=0.06$ and 0.04 , respectively) was independently associated with revival benefit in contrast to nondocumented patients [Table 4]; however, due to few number of patients, we could not interfere anything.

\begin{tabular}{|c|c|c|c|}
\hline \multirow[t]{2}{*}{ Component } & \multicolumn{2}{|c|}{$\begin{array}{l}\text { Compliance (percentage of CPR } \\
\text { documented) }\end{array}$} & \multirow[t]{2}{*}{$P$} \\
\hline & $\begin{array}{l}\text { Preintervention } \\
\qquad(n=40)\end{array}$ & $\begin{array}{l}\text { Postintervention } \\
\quad(n=86)\end{array}$ & \\
\hline Responsiveness & $26(65)$ & $67(77.9)$ & 0.19 \\
\hline Breathing & $15(37.5)$ & $50(58.1)$ & 0.03 \\
\hline Carotid pulsation & $25(62.5)$ & $68(79)$ & 0.05 \\
\hline Chest compressions & $8(20)$ & $34(39.5)$ & 0.04 \\
\hline Airway management & $25(62.5)$ & $71(82.5)$ & 0.02 \\
\hline $\begin{array}{l}\text { Compressions to } \\
\text { breaths ratio }\end{array}$ & $5(12.5)$ & $27(31.4)$ & 0.02 \\
\hline All & $1(2.5)$ & $13(15.11)$ & 0.03 \\
\hline
\end{tabular}

Figures in bracket indicate percentage. CPR: Cardiopulmonary resuscitation

\begin{tabular}{lccc}
\hline \multicolumn{3}{l}{$\begin{array}{l}\text { Table 3: Survival analysis comparison in-between } \\
\text { preintervention and postintervention groups }\end{array}$} & \\
\hline Component & $\begin{array}{c}\text { Preintervention } \\
(\boldsymbol{n}=\mathbf{4 0 )}\end{array}$ & $\begin{array}{c}\text { Postintervention } \\
(\boldsymbol{n = 8 6 )}\end{array}$ & $\boldsymbol{P}$ \\
& 17.5 & 18.6 & 1 \\
\hline Rate of ROSC (\%) & 10.6 & 12.6 & 0.4 \\
\hline
\end{tabular}

ROSC: Return of spontaneous circulation

Table 4: Comparison of cardiopulmonary resuscitation components among revived/return of spontaneous circulation patients

\begin{tabular}{lccc}
\hline Components & \multicolumn{2}{c}{$\begin{array}{c}\text { Post-CPR rate of ROSC } \\
(\boldsymbol{n}=\mathbf{2 3})\end{array}$} & $P$ \\
\cline { 2 - 3 } & Documented & $\begin{array}{c}\text { Not } \\
\text { documented }\end{array}$ \\
\hline Responsiveness & $21(96)$ & $2(33)$ & 0.06 \\
Breathing & $15(65)$ & $8(61)$ & 0.17 \\
Carotid pulse & $19(93)$ & $4(33)$ & 0.43 \\
Chest compressions rate & $12(42)$ & $11(84)$ & 0.04 \\
Airway management & $18(9)$ & $5(30)$ & 1.0 \\
Ratio of compressions & $8(32)$ & $15(94)$ & 0.29 \\
to breaths & & & \\
All & $5(14)$ & $18(112)$ & 0.13 \\
\hline
\end{tabular}

Figures in bracket indicate total number of documented and not documented patients, respectively. ROSC: Return of spontaneous circulation; CPR: Cardiopulmonary resuscitation

\section{Discussion}

This QI study, the first of this kind, involved in-depth documentation evaluation of CPR components before and after resident training with the aim of assessing AHA-2010 CPR guideline compliance. The study established that CPR providers had poor baseline CPR guideline compliance of documentation. This compliance improved significantly by a focused and brief CPR training intervention directed at the aforementioned CPR providers as shown in the improvement of documentation quality. One may have skill/knowledge in CPR procedure as per the guidelines, but adequate documentation proves effective use of it or to be compliant. Although simulated training programs or other methods improve skills in CPR (as proved by many other studies), no previous study has determined the assessment of CPR guideline compliance.$^{[15,18-20]}$ Hence, this may be the first study to assess indirectly the guideline compliance by seeing the documentation content and quality. However, outcomes of CPR did not improve significantly, perhaps due to lack of control over other factors involved in the system of care.

Observation of live CPR procedure to determine accuracy/ compliance without any participation of the observer in the resuscitative effort itself is ethically unsound. Documentation review is an alternative method to assess the guideline compliance. Another indirect method, survival analysis, may also be used as a surrogate as poor-quality CPR will ultimately result in poor short-and long-term outcomes. However, bad outcome does not mean noncompliant CPR since outcome also depends on the primary cause of arrest. For example, patients of sepsis who suffer cardiac arrest are more likely to have worse outcomes than those with a purely cardiac cause of arrest. ${ }^{[21]}$

Several recent studies highlight the fact that the CPR benefits increase as the number of completed components increase. ${ }^{[5,6,22,23]}$ There are only sparse data regarding compliance to the documentations of CPR components in Indian hospitals and even internationally due to the inherent risks involved in publishing such data. Western hospitals use internal quality control processes to assess this and accordingly take necessary action if low compliance is found; however, Indian hospitals do not have such QI programs. Our study showed the improvement of postintervention documentations of four components of CPR, viz., assessment of breathing, rate of chest compressions, airway maintenance, and compressions to breaths ratio, to be statistically significant $(P \leq 0.05)$. Furthermore, considering all components simultaneously in a patient, the compliance was low. The reasons for this may be due to busy Indian emergency schedules, forgetfulness of documenting all components in the CPR progress note, training issues, and most importantly human behavior-related factors; not all these were studied in this study. Other components including detection of rhythm and use of defibrillation are assessed, but they lose statistical significance. Recent recommendations for high-quality CPR include a simultaneous, choreographed approach to the performance of chest compressions, airway 
management, rescue breathing, rhythm detection, and shock delivery (if indicated) by an integrated team of highly trained rescuers in applicable settings. Few do's and don'ts are also advocated [Table 5]. Hence, every institute should prepare their own components of CPR as resident provider is concerned in this whole QI process.

Indian studies have shown that in-hospital revival/ROSC in patients requiring CPR is $18.4 \%$ and survival to discharge is $14.4 \%$, similar to the world population having revival rate of $19 \% .^{[24,25]}$ In this small sample study, ROSC is comparable but without any significant improvement from $17.5 \%$ to $18.6 \%$ postintervention. This may be due to lack of control over other QI factors that were not considered in this study. Survival to hospital discharge is also much lower in our study. The mean hospital stay, which is also an indirect marker of CPR guideline compliance, increased postintervention only by 2 days but has same consequence like ROSC. This shows poor post-CPR quality of life care and may signify lack of adequate ICU care which is in reality an important barrier in many Indian hospitals. In subgroup analysis of ROSC patients, there is a statistical increase in chance of revival in case of documentations of rate of chest compressions $(P=0.04)$ only. Other components may have been applied, but not documented properly showing the casual attitude toward writing a CPR progress note. Furthermore, certain confounding factors could not be eliminated such as elderly or terminal patients receiving inadequate CPR.

For a CPR training program to be successful, it should be convenient, relevant, focused, and delivered to the target population. We had targeted the relevant population (resident physicians) which constituted those most likely to respond to an in-hospital cardiac arrest before the arrival of trained critical care providers and those most likely to document CPR notes at the end. The individual duties of team members during CPR may vary, but the resident is the team leader and backbone of this life-saving process. Henceforth, these facts reinforce the strength of the study design and relevance

\begin{tabular}{ll}
\hline $\begin{array}{l}\text { Table 5: Basic life support do's and don'ts of adult } \\
\text { high-quality cardiopulmonary resuscitation }\end{array}$ \\
\hline Rescuers should not & Rescuers should \\
\hline $\begin{array}{l}\text { Compress at a rate slower } \\
\text { than } 100 / \text { min or faster } \\
\text { than } 120 / \text { min }\end{array}$ & $\begin{array}{l}\text { Perform chest compressions at a rate } \\
\text { of } 100-120 / \text { min }\end{array}$ \\
$\begin{array}{l}\text { Compress to a depth } \\
\text { of }<2 \text { inches ( } 5 \text { cm) or }\end{array}$ & $\begin{array}{l}\text { Compress to a depth of at least } \\
>2.4 \text { inches ( } 6 \text { cm) }\end{array}$ \\
$\begin{array}{l}\text { Lean on the chest } \\
\text { between compressions }\end{array}$ & $\begin{array}{l}\text { Allow full recoil after each } \\
\text { compression }\end{array}$ \\
$\begin{array}{l}\text { Interrupt compressions } \\
\text { for }>10 \mathrm{~s}\end{array}$ & Minimize pauses in compressions \\
$\begin{array}{l}\text { Provide excessive } \\
\text { ventilation (i.e., too many } \\
\text { breaths or breaths with } \\
\text { excessive force) }\end{array}$ & $\begin{array}{l}\text { Ventilate adequately ( } 2 \text { breaths } \\
\text { delivered over } 1 \mathrm{~s} \text {, each causing }\end{array}$ \\
\hline
\end{tabular}

to the resuscitation education. Furthermore, we observed other obstacles in providing quality CPR including lack of adequate workforce (resident doctor was the only provider many-a-times), delay in response time by medical personnel in an overcrowded Indian hospital setting, practical limitations in implementing training programs, difficulty in monitoring CPR in a busy emergency ward, and lack of appropriate means and devices for continuous and accurate surveillance of compliance to CPR guidelines. Our institute continues to work toward meeting goals set for this QI program and overcoming barrier.

This QI study has notable limitations. First, the durability of improvement (skill retention) is a major question as this will determine the frequency at which doctors should be re-educated. Although recent guidelines re-enforce the importance of periodic refresher courses in CPR, none have recommended the optimum time interval for recertification of basic and advanced life support programs. ${ }^{\left[{ }^{[9}\right.}$ Second, our study participants were medicine residents only; therefore, it is difficult to generalize our findings to other care providers. However, the success of this program is most likely attributable to its focus on CPR technique and proper documentation of the CPR process, which should be independent of medical specialty and applicable to all hospital-based responders as well. Third, we did not measure other variables as discussed above including the contribution of other medical personnel, training of nursing staff, immediate availability of resuscitation tray, overburdened residents, their behavior, and other unknown factors. These may have blunted the improvement in guideline compliance posttraining (only 12.39\%). Although individual components have $>50 \%$ compliance, chest compression rate and chest compressions to breaths ratio remained very low. This shows poor documenting quality of human behavioral aspect. CPR procedure is a psychosomatic skill; therefore, in all future efforts to determine guideline compliance, behavioral assessment should be included as well. Identifying and rectifying all these variables could have led to a better rate of compliance in the study. Henceforth, this study encourages having large QI study/program for each institute to guide adequate compliance to CPR.

\section{Conclusions}

The study establishes that the compliance to CPR documentation compliance is poor as assessed by CPR documentation content and quality, which improves after physician training, but not up to satisfactory level (100\%) that may be due to busy Indian hospital settings and human behavioral factors. Furthermore, this document checklist approach may be considered as an internal quality assessment method for CPR compliance. Each hospital/institute should have an internal QI program for determining CPR guideline compliance. Correct initial training is required followed by periodical re-education during the residency period. Future studies should assess the efficacy of these training sessions (i.e., skill retention), the various barriers of poor documentation compliance, and investigate whether CPR documentation can be used as a surrogate marker 
for CPR quality as a part of an institute's internal QI program, since live CPR assessment is an ethical issue.

Finally, the authors would like to point out that while there is great scope for improvement of CPR quality in India, the withholding of CPR in terminally ill patients (in accordance with their wishes) in whom curative intent is medically futile is an important consideration in delivering a better quality of overall care.

\section{Financial support and sponsorship}

Nil.

\section{Conflicts of interest}

There are no conflicts of interest.

\section{References}

1. Sasson C, Rogers MA, Dahl J, Kellermann AL. Predictors of survival from out-of-hospital cardiac arrest: A systematic review and meta-analysis. Circ Cardiovasc Qual Outcomes 2010;3:63-81.

2. Eisenberg MS, Mengert TJ. Cardiac resuscitation. N Engl J Med 2001;344:1304-13.

3. Rea TD, Crouthamel M, Eisenberg MS, Becker LJ, Lima AR. Temporal patterns in long-term survival after resuscitation from out-of-hospital cardiac arrest. Circulation 2003;108:1196-201.

4. Nolan JP, Hazinski MF, Billi JE, Boettiger BW, Bossaert L, de Caen AR, et al. Part 1: Executive summary: 2010 international consensus on cardiopulmonary resuscitation and emergency cardiovascular care science with treatment recommendations. Resuscitation 2010;81 Suppl 1:e1-25.

5. Van Hoeyweghen RJ, Bossaert LL, Mullie A, Calle P, Martens P, Buylaert WA, et al. Quality and efficiency of bystander CPR. Belgian Cerebral Resuscitation Study Group. Resuscitation 1993;26:47-52.

6. Gallagher EJ, Lombardi G, Gennis P. Effectiveness of bystander cardiopulmonary resuscitation and survival following out-of-hospital cardiac arrest. JAMA 1995;274:1922-5.

7. Cummins RO, Chesemore K, White RD. Defibrillator failures. Causes of problems and recommendations for improvement. Defibrillator Working Group. JAMA 1990;264:1019-25.

8. Kellermann AL, Hackman BB, Dobyns P, Frazier C, Nail L. Engineering excellence: Options to enhance firefighter compliance with standing orders for first-responder defibrillation. Ann Emerg Med 1993;22:1269-75.

9. Bhanji F, Donoghue AJ, Wolff MS, Flores GE, Halamek LP, Berman JM, et al. Part 14: Education: 2015 American heart Association guidelines update for cardiopulmonary resuscitation and emergency cardiovascular care. Circulation 2015;132:S561-73.

10. Kaye W, Mancini ME. Retention of cardiopulmonary resuscitation skills by physicians, registered nurses, and the general public. Crit Care Med 1986;14:620-2.

11. Wayne DB, Butter J, Siddall VJ, Fudala MJ, Linquist LA, Feinglass J, et al. Simulation-based training of internal medicine residents in advanced cardiac life support protocols: A randomized trial. Teach Learn Med 2005;17:210-6.

12. Wayne DB, Didwania A, Feinglass J, Fudala MJ, Barsuk JH, McGaghie WC, et al. Simulation-based education improves quality of care during cardiac arrest team responses at an academic teaching hospital: A case-control study. Chest 2008;133:56-61.

13. Yeung J, Meeks R, Edelson D, Gao F, Soar J, Perkins GD, et al. The use of CPR feedback/prompt devices during training and CPR performance: A systematic review. Resuscitation 2009;80:743-51.

14. Edelson DP, Litzinger B, Arora V, Walsh D, Kim S, Lauderdale DS, et al. Improving in-hospital cardiac arrest process and outcomes with performance debriefing. Arch Intern Med 2008;168:1063-9.

15. Sutton RM, Niles D, Meaney PA, Aplenc R, French B, Abella BS, et al. "Booster" training: Evaluation of instructor-led bedside cardiopulmonary resuscitation skill training and automated corrective feedback to improve cardiopulmonary resuscitation compliance of pediatric basic life support providers during simulated cardiac arrest. Pediatr Crit Care Med 2011;12:e116-21.

16. Dick WF. Uniform reporting in resuscitation. $\mathrm{Br} \mathrm{J}$ Anaesth 1997;79:241-52.

17. Berg RA, Hemphill R, Abella BS, Aufderheide TP, Cave DM, Hazinski MF, et al. Part 5: Adult basic life support: 2010 American Heart Association Guidelines for cardiopulmonary resuscitation and emergency cardiovascular care. Circulation 2010;122:S685-705.

18. Nurmi J, Pettilä V, Biber B, Kuisma M, Komulainen R, Castrén M, et al. Effect of protocol compliance to cardiac arrest identification by emergency medical dispatchers. Resuscitation 2006;70:463-9.

19. Lapostolle F, Le Toumelin P, Agostinucci JM, Catineau J, Adnet F. Basic cardiac life support providers checking the carotid pulse: Performance, degree of conviction, and influencing factors. Acad Emerg Med 2004;11:878-80

20. Thigpen K, Davis SP, Basol R, Lange P, Jain SS, Olsen JD, et al. Implementing the 2005 American Heart Association Guidelines, including use of the impedance threshold device, improves hospital discharge rate after in-hospital cardiac arrest. Respir Care 2010;55:1014-9.

21. Ebell MH, Becker LA, Barry HC, Hagen M. Survival after in-hospital cardiopulmonary resuscitation. A meta-analysis. J Gen Intern Med 1998;13:805-16.

22. Feneley MP, Maier GW, Kern KB, Gaynor JW, Gall SA Jr., Sanders AB, et al. Influence of compression rate on initial success of resuscitation and 24 hour survival after prolonged manual cardiopulmonary resuscitation in dogs. Circulation 1988;77:240-50.

23. Berg RA, Sanders AB, Kern KB, Hilwig RW, Heidenreich JW, Porter ME, et al. Adverse hemodynamic effects of interrupting chest compressions for rescue breathing during cardiopulmonary resuscitation for ventricular fibrillation cardiac arrest. Circulation 2001;104:2465-70.

24. Rajaram R, Rajagopalan RE, Pai M, Mahendran S. Survival after cardiopulmonary resuscitation in an urban Indian hospital. Natl Med J India 1999;12:51-5.

25. Writing Group Members, Lloyd-Jones D, Adams RJ, Brown TM, Carnethon M, Dai S, et al. Heart disease and stroke statistics-2010 update: A report from the American Heart Association. Circulation 2010;121:e46-215. 